\title{
THE BLASCHKE CONDITION FOR BOUNDED HOLOMORPHIC FUNCTIONS
}

\author{
PAK SOONG CHEE
}

1. Introduction. Let $U$ be the unit disc in the complex plane $C$ and $H^{\infty}(U)$ the space of all bounded holomorphic functions in $U$. Let $f \in H^{\infty}(U), f \neq 0$, and let $\alpha_{1}, \alpha_{2}, \ldots$ be the zeros of $f$, listed according to multiplicities. For $0<r<1$, let $n(r)$ be the number of $\alpha$ 's with $\left|\alpha_{n}\right| \leqq r$. Then it is well known that the Blaschke condition

$$
\int_{0}^{1} n(r) d r<\infty
$$

is satisfied. This is a consequence of Jensen's formula in the form:

$$
\int_{0}^{r} \frac{n(x)}{x} d x=\frac{1}{2 \pi} \int_{0}^{2 \pi} \log \left|f\left(r e^{i \theta}\right)\right| d \theta-\log |f(0)|
$$

(see e.g. $[11, \S 3.61])$.

The aim of the present paper is to study the generalization of (1) to several variables. We show that the $N$-dimensional volume (given in terms of the Hausdorff measure) of the zero-set $Z(f)$ of a bounded holomorphic function $f$ in the unit polydisc $U^{N+1}$ or the unit ball $B_{N+1}$ in $C^{N+1}$ satisfies the generalized condition (Theorem 6.3).

In one variable, the condition (1) is also sufficient for the set $\left\{\alpha_{n}\right\}$ to be the zeroset of a bounded holomorphic function in $U$ (see [7, Theorem 15.21]). For more than one variable, this is no longer so, and we give two examples in $§ 7$. In this direction, Professor Rudin was the first to obtain a global condition sufficient for a subvariety $E$ in $U^{N}$ to be the zero-set of a bounded holomorphic function, namely the condition dist $\left(E, T^{N}\right)>0$, where $T^{N}$ is the $N$-dimensional torus (see [6, Theorem 4.8.3]). Recently, Stout [10] has given a different set of sufficient conditions. No sufficient condition seems to be known in $B_{N}$.

This paper is part of the author's Ph.D. thesis at the University of Wisconsin. I wish to thank my advisor, Professor Walter Rudin, for his generous help and encouragement during the preparation of this work.

2. Hausdorff measures. A lemma on matrices. For our later applications, we summarize here the definition and some elementary properties of the Hausdorff measure.

Received by the editors June 22, 1969. 
Let $A$ be a subset of a metric space $X$. Let $\delta(A)$ denote the diameter of $A$. Write $\delta^{P}(A)=[\delta(A)]^{P}$ for $p>0 ; \delta^{0}(A)=1$ if $A \neq \varnothing$, and $\delta^{0}(\phi)=0$. For $p \geqq 0, \varepsilon>0$, define

$$
\begin{aligned}
H_{p}(A ; \varepsilon) & =\inf \left\{\sum_{n=1}^{\infty} \delta^{P}\left(A_{n}\right): A \subseteq \bigcup_{n=1}^{\infty} A_{n} \text { and } \delta\left(A_{n}\right)<\varepsilon\right\}, \\
H_{p}(A) & =\lim _{\varepsilon \rightarrow 0+} C_{p} H_{p}(A ; \varepsilon),
\end{aligned}
$$

where $C_{p}=\pi^{p / 2} /\left(2^{p} \Gamma(p / 2+1)\right)$.

If $N$ is an integer, then $C_{N}$ is the volume of the ball $\left\{x \in \boldsymbol{R}^{N}: \sum_{1}^{N} x_{i}^{2} \leqq \frac{1}{4}\right\} . H_{p}(A)$ is called the $p$-dimensional Hausdorff measure or the Hausdorff $p$-measure of $A$. For any $A \subseteq X, H_{0}(A)$ equals the number of points in $A$.

For any $p \geqq 0, H_{p}$ is a regular metric outer measure and hence the Borel sets are $H_{p}$-measurable (see [5, §12]). The Hausdorff measures have the following important elementary properties:

(i) If $H_{p}(A)<\infty$ and $r>p$, then $H_{r}(A)=0$; hence if $A$ is $H_{p}-\sigma$-finite, then $H_{r}(A)$ $=0$.

(ii) Let $Y$ be a metric space and $f: X \rightarrow Y$ be a Lipschitz map with Lipschitz constant $\lambda$. Then for any $A \subseteq X$ and any $p \geqq 0, H_{p}(f(A)) \leqq \lambda^{p} H_{p}(A)$.

(iii) If $M$ is a $k$-dimensional $C^{1}$ submanifold of $R^{N}$, then volume of $M=H_{k}(M)$.

The first two properties follow directly from definitions; for (iii), see Stolzenberg [9].

It follows from (i) that the singular locus $S$ of a pure $k$-dimensional analytic subvariety in $C^{N}$ has Hausdorff $2 k$-measure zero, since $S$ is the countable union of manifolds of real dimension at most $2(k-1)$. By (iii) we see that the Lebesgue measure on $\boldsymbol{R}^{N}$ is equal to the Hausdorff $N$-measure on $\boldsymbol{R}^{N}$.

We insert here a lemma on matrices which must be well known. We include a proof for lack of a suitable reference. First, some definitions.

Let $A=\left(a_{m j}\right)$ be any complex matrix with $n$ rows and $k$ columns (an $n \times k$ matrix). Let $a_{m j}=b_{m j}+i c_{m j}$. Then $\tilde{A}$ will denote the real $2 n \times 2 k$ matrix obtained from $A$ by replacing each $a_{m j}$ by the $2 \times 2$ matrix

$$
\left(\begin{array}{rr}
b_{m j} & c_{m j} \\
-c_{m j} & b_{m j}
\end{array}\right) .
$$

It is easy to check that

(i) $(A+B)^{\sim}=\tilde{A}+\widetilde{B}$,

(ii) $\left(\bar{A}^{\prime}\right)^{\sim}=\bar{A}^{\prime}$, where $\bar{A}=\left(\bar{a}_{m j}\right)$,

(iii) $(A B)^{\sim}=\tilde{A} \widetilde{B}$, whenever the product $A B$ is defined,

(iv) $U$ is unitary implies $\tilde{U}$ is orthogonal.

The first three statements can be verified by writing out both sides. The fourth follows from (ii) and (iii). 
Let $k \leqq n$, and let $A$ be any complex $n \times k$ matrix. Define

$$
\begin{aligned}
& \Delta(A)=\sum_{x}\left|\operatorname{det} A_{x}\right|^{2}, \\
& \tilde{\Delta}(\tilde{A})=\left\{\sum_{y}\left|\operatorname{det} \tilde{A_{y}}\right|^{2}\right\}^{1 / 2},
\end{aligned}
$$

where $A_{x}$ runs over all $k \times k$ submatrices of $A$ and $\tilde{A}_{y}$ runs over all $2 k \times 2 k$ submatrices of $\tilde{A}$.

If $A$ and $B$ are two $n \times n$ matrices, then $\operatorname{det}(B A)=\operatorname{det} B \cdot \operatorname{det} A$. This has the following generalization.

CAuChy-Binet Theorem. Let $k \leqq n$. Let $A$ be an $n \times k$ matrix and $B$ a $k \times n$ matrix. Then $\operatorname{det}(B A)$ is equal to the sum of all the $\left(\begin{array}{c}n \\ k\end{array}\right)$ products which can be made by taking a minor of order $k$ from certain $k$ columns of $B$ and a minor of order $k$ from the corresponding rows of $A$. (See Aitken $[1, \S 36]$.)

Lemma 2.1. Let $A$ be any complex $n \times k$ matrix, $k \leqq n$. Then $\Delta(A)=\tilde{\Delta}(\tilde{A})$.

Proof. Let $A^{\prime}$ denote the transpose of $A$. Then by the Cauchy-Binet Theorem,

$$
\Delta(A)=\operatorname{det}\left(\bar{A}^{\prime} A\right), \quad \tilde{\Delta}(\tilde{A})=\left\{\operatorname{det}\left(\tilde{A}^{\prime} \tilde{A}\right)\right\}^{1 / 2} .
$$

Therefore if $U$ is a unitary $n \times n$ matrix, and $\tilde{U}$ the associated orthogonal matrix, then

$$
\Delta(A)=\Delta(U A), \quad \tilde{\Delta}(\tilde{A})=\tilde{\Delta}(\tilde{U} \tilde{A}) .
$$

Given $A$, we choose a unitary $n \times n$ matrix $U$ such that the bottom $(n-k)$ rows of $U$ are orthogonal to each of the $k$ columns of $A$. Then the bottom $(n-k)$ rows of $U A$ are zero. Let $B$ be the $k \times k$ submatrix of $U A$ formed by the first $k$ rows. Then

$$
\Delta(A)=\Delta(U A)=|\operatorname{det} B|^{2} .
$$

Now $\tilde{U} \tilde{A}=(U A)^{\sim}$ has the bottom $2(n-k)$ rows equal to zero and the $2 k \times 2 k$ submatrix formed by the first $2 k$ rows of $\tilde{U} \tilde{A}$ is $\widetilde{B}$. Therefore

$$
\tilde{\Delta}(\tilde{A})=\tilde{\Delta}(\tilde{U} \tilde{A})=|\operatorname{det} \tilde{B}| .
$$

It remains to show that $|\operatorname{det} \widetilde{B}|=|\operatorname{det} B|^{2}$.

Assume first that $B$ has $k$ distinct eigenvalues $\lambda_{1}, \ldots, \lambda_{k}$ and that $\lambda_{i} \neq \bar{\lambda}_{j}$ for all $i, j$. To each $\lambda_{j}$ corresponds an eigenvector $\left(z_{1}, \ldots, z_{k}\right)$ of $B$. It is easy to verify that then $\left(z_{1}, i z_{1}, \ldots, z_{k}, i z_{k}\right)$ is an eigenvector of $\widetilde{B}$ with the same eigenvalue $\lambda_{j}$. Since $\widetilde{B}$ is real $\bar{\lambda}_{j}$ is also an eigenvalue of $\widetilde{B}$. Thus $\lambda_{1}, \lambda_{1}, \ldots, \lambda_{k}, \bar{\lambda}_{k}$ are $2 k$ distinct eigenvalues of $\widetilde{B}$, so there are no others. Hence

$$
\operatorname{det} \tilde{B}=\lambda_{1} \bar{\lambda}_{1} \cdots \lambda_{k} \bar{\lambda}_{k}=\left|\lambda_{1} \cdots \lambda_{k}\right|^{2}=|\operatorname{det} B|^{2} .
$$

The general case follows by continuity. 
3. The volume of an analytic variety. A pure $k$-dimensional analytic subvariety in $C^{N}$ is the closure of a complex $k$-dimensional manifold, viz., the set of its regular points. A complex analytic manifold is also a real analytic manifold. To get a formula for the volume of an analytic subvariety, we shall use the following facts concerning the volume of manifolds in $\boldsymbol{R}^{N}$. For an account of these, see Schwartz [8, Chapter IV, §10].

Let $M$ be an open subset of a $C^{1}$ submanifold of dimension $k$ in $R^{N}$. Suppose $M$ is homeomorphic to an open subset $\Omega$ in $\boldsymbol{R}^{k}$ under the map $\Phi: \Omega \rightarrow M$, where $\Phi$ and its inverse $\Phi^{-1}$ are both of class $C^{1}$. Let $L$ be the Jacobian matrix of $\Phi$. Define $J \Phi=(\Delta(L))^{1 / 2}$ where $\Delta(L)$ is given by formula (5). Then by Theorem 107 of [8, p. 688], the $k$-dimensional volume of $M$ is given by

$$
H_{k}(M)=\int_{\Omega} J \Phi(x) d H_{k}(x) .
$$

THEOREM 3.1. Let $\Omega$ be an open subset of $\boldsymbol{R}^{k}$ and let $\Phi: \Omega \rightarrow \boldsymbol{R}^{N}$ be a $C^{1}$ map from $\Omega$ into $\boldsymbol{R}^{N}$. Then for any Lebesgue measurable subset $A$ of $\Omega$,

$$
\int_{\Phi(A)} H_{0}\left\{A \cap \Phi^{-1}(y)\right\} d H_{k}(y)=\int_{A} J \Phi(x) d H_{k}(x) .
$$

This is proved in Federer [3, Theorem 4.5], for Lipschitz maps and the measure $\mathscr{L}_{N}^{k}$. The same proof works for $C^{1}$ maps and Hausdorff measures.

Now let $V$ be a pure $k$-dimensional analytic subvariety in a domain $\Omega$ in $C^{N}$. Let $S$ be the singular locus of $V$. Then as we have seen $H_{2 k}(S)=0$ and so $H_{2 k}(V)$ $=H_{2 k}(V-S)$. We shall establish the following integral geometric formula for the volume of an analytic variety:

THEOREM 3.2. Let $V$ be a pure (complex) $k$-dimensional analytic subvariety in $a$ domain $\Omega$ in $C^{N}$. Let the $k$-dimensional coordinate subspaces of $C^{N}$ be enumerated in some order. Let $\pi_{j}$ be the projection from $C^{N}$ onto the jth subspace and write $\hat{z}_{j}=\pi_{j}(z)$. Then

$$
H_{2 k}(V)=\sum_{j=1}^{m} \int_{\pi_{j} \Omega} H_{0}\left\{V \cap \pi_{j}^{-1}\left(\hat{z}_{j}\right)\right\} d H_{2 k}\left(\hat{z}_{j}\right)
$$

where $m=\left(\begin{array}{l}N \\ k\end{array}\right)$.

Proof. Let $R=V-S$ be the set of regular points of $V$. We shall show that (9) holds for $R$. Since both sides of (9) are regular Borel measures, it is sufficient to show that it holds for $R \cap K, K$ any compact subset of $\Omega$.

For each $z \in R$, we can find a neighborhood $B$ of arbitrarily small diameter which is holomorphically homeomorphic to a closed polydisc $A$ in $C^{k}$. The sets $B \cap K$ form a covering of $R \cap K$ in the sense of Vitali. By the classical covering theorem of Vitali, there exists a countable disjoint family $\left\{B_{j} \cap K\right\}_{1}^{\infty}$ such that

$$
H_{2 k}\left(R \cap K-\bigcup_{1}^{\infty} B_{j} \cap K\right)=0
$$

Hence it suffices to prove the formula (9) for each set $B_{j} \cap K$. 
Thus let $B$ be a subset of $R$ which is holomorphically homeomorphic to a closed polydisc $A$ in $C^{k}$, under the map

$$
F: A \rightarrow B, \quad F(x)=\left(f_{1}(x), \ldots, f_{N}(x)\right) .
$$

Let $L$ be the complex Jacobian matrix of $F$ and $\tilde{L}$ the associated real Jacobian matrix. Let $J F=\Delta(L)$ and $\tilde{J} F=\tilde{\Delta}(\tilde{L})$ as given by (5) and (6). Then by (7),

$$
H_{2 k}(B)=\int_{A} \tilde{J} F(x) d H_{2 k}(x) .
$$

This holds since the boundary of $A$ has measure zero and so does its image, the boundary of $B$, on account of property (ii) of the Hausdorff measures. By Lemma $2.1, \tilde{J} F=J F$ and so

$$
H_{2 k}(B)=\int_{A} J F(x) d H_{2 k}(x)=\sum_{j=1}^{m} \int_{A}\left|\operatorname{det} L_{j}(x)\right|^{2} d H_{2 k}(x),
$$

where the $L_{j}$ 's are the $k \times k$ submatrices of $L$. With $\pi_{j}$ as defined in the statement of the theorem, each $L_{j}$ can be regarded as the Jacobian matrix of the map $F_{j}=$ $\pi_{j} \circ F: A \rightarrow C^{N}$ mapping $A$ into the $j$ th $k$-dimensional coordinate subspace of $C^{N}$. $F$ being a homeomorphism implies

$$
H_{0}\left\{A \cap F_{j}^{-1}\left(\hat{z}_{j}\right)\right\}=H_{0}\left\{B \cap \pi_{j}^{-1}\left(\hat{z}_{j}\right)\right\} .
$$

So since $J F_{j}=\left|\operatorname{det} L_{j}\right|^{2}$, Theorem 3.1, formula (8) gives

$$
\int_{A}\left|\operatorname{det} L_{j}(x)\right|^{2} d H_{2 k}(x)=\int_{\pi_{j} B} H_{0}\left\{B \cap \pi_{j}^{-1}\left(\hat{z}_{j}\right)\right\} d H_{2 k}\left(\hat{z}_{j}\right) .
$$

Substituting in (10) we get

$$
H_{2 k}(B)=\sum_{j=1}^{m} \int_{\pi_{j} B} H_{0}\left\{B \cap \pi_{j}^{-1}\left(\hat{z}_{j}\right)\right\} d H_{2 k}\left(\hat{z}_{j}\right) .
$$

Noting that $H_{0}\left\{B \cap \pi_{j}^{-1}\left(\hat{z}_{j}\right)\right\}=0$ if $\hat{z}_{j} \notin \pi_{j} B$, we may replace the domain of integration $\pi_{j} B$ by $\pi_{j} \Omega$ and so complete the proof of (9) for the set $B$.

For later application, we give here a generalization of (9):

THEOREM 3.3. With the notation as in Theorem 3.2, let $f$ be a nonnegative Borel function which vanishes outside $V$. Then

$$
\int_{\Omega} f(z) d H_{2 k}(z)=\sum_{j=1}^{m} \int_{\pi_{j} \Omega^{2}} d H_{2 k}\left(\hat{z}_{j}\right) \int_{\pi_{j}^{-1}\left(\hat{z}_{j}\right)} f(z) d H_{0}\left(z_{j}\right) .
$$

Proof. The proof of Theorem 3.2 shows that (9) holds for any open subset of $V$. Hence since both sides of (9) are regular Borel measures, it holds for any Borel subset $A$ of $V$. Thus (11) holds if $f=\chi_{A}$, the characteristic function of $A$; hence it holds if $f$ is any nonnegative simple Borel function vanishing outside $V$. If $f$ is any nonnegative Borel function, then there is an increasing sequence of nonnegative 
simple Borel functions $s_{n}$ such that $\lim s_{n}=f$. Since (11) holds for each $s_{n}$, the monotone convergence theorem shows that it holds for $f$.

4. The multiplicity function. Let $f$ be a holomorphic function in a domain $\Omega$ in $C^{N}$. For each $a \in \Omega$, we define the zero-multiplicity $\mu(a)=\mu_{f}(a)$ of $f$ at $a$ as follows: If $f \equiv 0$, then $\mu(a)=\infty$. If $f \not \equiv 0$, then $f$ has an expansion of the form

$$
f(z)=f_{m}(z-a)+f_{m+1}(z-a)+\cdots
$$

in a neighborhood of $a$, where $f_{j}$ is a homogeneous polynomial of degree $j$ and $f_{m} \not \equiv 0$. Define $\mu(a)=m$.

The following observations can be made:

(i) If $f=g h$, then $\mu_{f}=\mu_{g}+\mu_{h}$.

(ii) $\mu(a)$ does not depend on the choice of coordinates at $a$.

(iii) If $0 \in \Omega$ and $\mu(0)=m>0$, then by the Weierstrass preparation theorem, there is a coordinate system $z_{1}, \ldots, z_{N}$ such that

$$
f=u W
$$

in a neighborhood of 0 , where $u$ has no zeros in that neighborhood and $W$ is a Weierstrass polynomial of degree $m$ in $z_{N}$.

(iv) The converse of (iii) is also true, viz., if (12) holds, then $\mu(0)=m$, the degree of $W$ in $z_{N}$.

The first two observations follow easily from definition (see $[6,1.1 .6]$ ); (iv) is a consequence of the uniqueness of the Weierstrass polynomial for $f$.

Proposition 4.1. Let $f$ be a holomorphic function in a domain $\Omega$ in $C^{N}, N>1$. Then $\mu$ is constant on each (connectivity) component of the set of regular points of $V=Z(f)$.

Proof. Without loss of generality, let 0 be a regular point of $V$. Let $\mu(0)=m$. If $m=\infty$, then $f \equiv 0$ and the proposition is trivial. Suppose $m<\infty$. At a regular point, $V$ is an $(N-1)$-dimensional manifold. By the definition of a manifold (see [4, I.B. 8]), there exist a neighborhood $U_{0}$ of 0 and a mapping $F: U_{0} \rightarrow C$ which is nonsingular at 0 such that $V \cap U_{0}=Z(F) . F$ being nonsingular at 0 implies that $\partial F(0) / \partial z_{j} \neq 0$ for some $j, 1 \leqq j \leqq N$. By renaming the coordinates, we may assume that $j=N$ and $\partial F(0) / \partial z_{N} \neq 0$. By the implicit function theorem, there is a disc $D$ containing $0 \in C$ and there is a function $\varphi\left(z^{\prime}\right)$ holomorphic in $D^{N-1}$ such that $\left(z^{\prime}, z_{N}\right) \in D^{N-1} \times D$ and $f\left(z^{\prime}, z_{N}\right)=0$ if and only if $z_{N}=\varphi\left(z^{\prime}\right)$. Define $p(z)=z_{N}-\varphi\left(z^{\prime}\right)$, $z \in D^{N}$. Then in a possibly smaller neighborhood of $0 \in C^{N}$ (again denoted by $U_{0}$ ) we have

$$
Z(f) \cap U_{0}=Z(F) \cap U_{0}=Z(p) \cap U_{0} .
$$

Thus in $U_{0}, f(z)=0$ if and only if $p(z)=0$. Since $p\left(0^{\prime}, z_{N}\right) \not \equiv 0, f\left(0^{\prime}, z_{N}\right) \not \equiv 0$. Therefore by the Weierstrass preparation theorem, $f=u W$ in a neighborhood $U_{1}$ of 0 , where $u(z) \neq 0$ for all $z \in U_{1}$ and $W$ is a Weierstrass polynomial of degree $m$ in $z_{N}$. Let 
$W=\prod_{i=1}^{s} p_{i}^{n_{i}}$ be the factorization of $W$ into irreducible factors. By taking $U_{1}$ small enough, we may assume that each $p_{i}$ is holomorphic in $U_{1}$ and $U_{1} \subseteq U_{0}$. Then

$$
Z(p) \cap U_{1}=Z(f) \cap U_{1}=Z(W) \cap U_{1}=\bigcup_{i=1}^{s} Z\left(p_{i}\right) .
$$

Since $Z(p)$ is an irreducible variety at 0 , the uniqueness of the irreducible decomposition of analytic varieties shows that $s=1$ and $p_{1}=p$. Thus $W=p^{n_{1}}$. Since $W$ is of degree $m$ and $p$ is of degree 1 in $z_{N}$, we must have $n_{1}=m$. So $f=u p^{m}$ in $U_{1}$.

Now let $b \in U_{1} \cap V$. We claim that $\mu(b)=m$. Let $w=z-b, z \in U_{1}$. Then $f(z)$ $=u(z)[p(z)]^{m}=\tilde{u}(w)[\tilde{p}(w)]^{m}$, where

$$
\tilde{p}(w)=p(w+b)=w_{N}+b_{N}-\varphi\left(w^{\prime}+b^{\prime}\right)=w_{N}+\tilde{\varphi}\left(w^{\prime}\right) .
$$

Since $b \in V, \tilde{\varphi}\left(0^{\prime}\right)=b_{N}-\varphi\left(b^{\prime}\right)=0$. Therefore $\tilde{p}$ is a Weierstrass polynomial at $b$, hence so is $\tilde{p}^{m}$. Since $f=\tilde{u} \tilde{p}^{m}$, the observation (iv) above shows that $\mu(b)=m$.

It follows then that $\mu$ is constant on each connected component of the set of regular points of $V$. Q.E.D.

This shows that the restriction of $\mu$ to the regular points of $V$ is a continuous function. In general we have

Proposition 4.2. Let $f$ be a holomorphic function in a domain $\Omega$ in $C^{N}$. Then its multiplicity function $\mu$ is upper semicontinuous in $\Omega$.

Proof. In the definition of $\mu$, the homogeneous polynomials $f_{j}$ are obtained by rearrangement of the Taylor series of $f$ at $a$. Thus if $f$ has a partial derivative of total order $n$ which is different from zero at $a$, then $\mu(a) \leqq n$. Now let $a \in \Omega$ and $\mu(a)=m$. Then $f$ has a partial derivative of total order $m$ different from zero at $a$. By the continuity of the partial derivative, it is different from zero in a neighborhood $U$ of $a$. So for any $b \in U, \mu(b) \leqq m$.

Combining this with Theorem 3.3, noting that the zero-set of a nontrivial holomorphic function is a pure dimensional subvariety of codimension one, we get

Corollary 4.3. Let $f$ be a holomorphic function in a domain $\Omega$ in $C^{N+1}, f \equiv 0$. Let $\mu$ be its multiplicity function. Then

$$
\int_{\Omega} \mu(z) d H_{2 N}(z)=\sum_{j=1}^{N+1} \int_{\pi_{j} \Omega} d H_{2 N}\left(\hat{z}_{j}\right) \int_{\pi_{j}^{-1}\left(\hat{z}_{j}\right)} \mu(z) d H_{0}\left(z_{j}\right)
$$

where $\pi_{j}: \boldsymbol{C}^{N+1} \rightarrow \boldsymbol{C}^{N+1}, \pi_{j}(z)=\left(z_{1}, \ldots, z_{j-1}, 0, z_{j+1}, \ldots, z_{N+1}\right) \equiv \hat{z}_{j}$.

5. The mean value of a plurisubharmonic function. We recall the definition of a plurisubharmonic function: Let $\Omega$ be a domain in $C^{N}$. A function $u: \Omega \rightarrow[-\infty, \infty)$ is called plurisubharmonic if

(i) $u$ is upper semicontinuous,

(ii) for any $z$ and $w \in C^{N}$, the function $\lambda \rightarrow u(z+\lambda w)$ is subharmonic where it is defined. 
For subharmonic functions, the following is well known.

THEOREM 5.1. Let $u$ be a subharmonic function in the unit disc $U, u \neq-\infty$. Let $m_{1}$ be the Lebesgue measure on $T$ normalized so that $m_{1}(T)=1$. Let

$$
M_{1}(r)=\int_{T} u(r \lambda) d m_{1}(\lambda), \quad 0 \leqq r<1 .
$$

Then (i) $M_{1}(r)>-\infty$ if $r>0$,

(ii) $M_{1}(r) \leqq M_{1}(s)$ if $r \leqq s$,

(iii) $M_{1}(r)$ is a convex function of $\log r$ in the interval $(0,1)$, i.e. $M_{1}\left(r_{1}^{a} r_{2}^{1-\alpha}\right)$ $\leqq \alpha M_{1}\left(r_{1}\right)+(1-\alpha) M_{1}\left(r_{2}\right)$, whenever $0 \leqq \alpha \leqq 1,0<r_{1} \leqq r_{2}<1$.

See [7, Chapter 17] and [12, Chapter 2]. This has been generalized to plurisubharmonic functions (see [12]). We give here a further generalization.

Let $\Omega$ be a domain in $C^{N}$. Then $\Omega$ is called a complete circular domain if $z=$ $\left(z_{1}, \ldots, z_{N}\right) \in \Omega$ and $|\lambda| \leqq 1$ imply $\lambda z=\left(\lambda z_{1}, \ldots, \lambda z_{N}\right) \in \Omega$. Thus $U^{N}$ and $B_{N}$ are complete circular domains. A measure $m$ on $\Omega$ is said to be circularly invariant if $m(E)=m\left(E_{\lambda}\right)$ for any measurable subset $E$ of $\Omega$ and any $\lambda \in T$, where $E_{\lambda}=$ $\{\lambda z: z \in E\}$. The Lebesgue measure is circularly invariant. Such measures were first considered by Bochner [2]. His method can be applied to prove the following.

THEOREM 5.2. Let $\Omega$ be a bounded complete circular domain in $C^{N}$. Let $m$ be a positive circularly invariant measure on $\Omega$ normalized so that $m(\Omega)=1$. Let $u$ be a plurisubharmonic function in $\Omega, u \neq \equiv$, and let

$$
M(r)=\int_{\Omega} u(r z) d m(z), \quad 0 \leqq r \leqq 1 .
$$

Then (i) $M(r)>-\infty$ if $r>0$ and $m$ is the Lebesgue measure,

(ii) $M(r) \leqq M(s)$ if $r \leqq s$,

(iii) $M(r)$ is a convex function of $\log r$ in the interval $(0,1)$.

Proof. To prove (ii) and (iii), we define for each $z \in \Omega, u_{z}(\lambda)=u(\lambda z)$. Then $u_{z}$ is a subharmonic function in a neighborhood of $\bar{U}$. So by Theorem 5.1, we have for all $z \in \Omega$ and $0 \leqq r \leqq s \leqq 1$,

$$
\int_{T} u(r \lambda z) d m_{1}(\lambda) \leqq \int_{T} u(s \lambda z) d m_{1}(\lambda)
$$

Since $m$ is circularly invariant,

$$
\int_{\Omega} u(r z) d m(z)=\int_{\Omega} u(r \lambda z) d m(z) \text { for all } \lambda \in T .
$$

Hence integrating over $T$, we get

$$
\int_{\Omega} u(r z) d m(z)=\int_{T} d m_{1}(\lambda) \int_{\Omega} u(r \lambda z) d m(z)=\int_{\Omega} d m(z) \int_{T} u(r \lambda z) d m_{1}(\lambda)
$$

by Fubini's theorem. 
From (14), we get by integrating over $\Omega$,

$$
\int_{\Omega} d m(z) \int_{T} u(r \lambda z) d m_{1}(\lambda) \leqq \int_{\Omega} d m(z) \int_{T} u(x \lambda z) d m_{1}(\lambda) .
$$

Hence substitution of (15) gives

$$
\int_{\Omega} u(r z) d m(z) \leqq \int_{\Omega} u(s z) d m(z), \quad 0 \leqq r \leqq s \leqq 1,
$$

which is (ii).

Let $M_{z}(r)=\int_{T} u(r \lambda z) d m_{1}(\lambda), z \in \Omega$. Then

$$
M_{z}\left(r_{1}^{\alpha} r_{2}^{1-\alpha}\right) \leqq \alpha M_{z}\left(r_{1}\right)+(1-\alpha) M_{z}\left(r_{2}\right)
$$

whenever $0 \leqq \alpha \leqq 1,0<r_{1} \leqq r_{2}<1$. Integrating over $\Omega$ and noting that by (15), $M(r)=\int_{\Omega} M_{z}(r) d m(z)$, we get

$$
M\left(r_{1}^{\alpha} r_{2}^{1-\alpha}\right) \leqq \alpha M\left(r_{1}\right)+(1-\alpha) M\left(r_{2}\right) .
$$

This proves (iii).

To prove (i), let $m$ be the Lebesgue measure on $\Omega$. Then since the Jacobian of the transformation $z \rightarrow r z$ is $r^{2 N}$,

$$
M(r)=\frac{1}{r^{2 N}} \int_{r \Omega} u(z) d m(z) .
$$

We note further that the proof of (ii) gives the following: If the ball $B=B(a, r)$ of center $a$ and radius $r$ is contained in $\Omega$, then

$$
u(a) \leqq \frac{1}{m(B)} \int_{B} u(z) d m(z) .
$$

Now suppose $M\left(r_{0}\right)=-\infty$ for some $r_{0}>0$. Then there exist an $a \in r_{0} \Omega$ and a number $r_{1}>0$ such that $B\left(a, 3 r_{1}\right) \subseteq \Omega$ and

$$
\int_{B\left(a, r_{1}\right)} u(z) d m(z)=-\infty
$$

By (16), $u(a)=-\infty$. Let $z^{\prime} \in B\left(a, r_{1}\right)$. Then

Therefore

$$
B\left(a, r_{1}\right) \subseteq B\left(z^{\prime}, 2 r_{1}\right) \subseteq B\left(a, 3 r_{1}\right)
$$

$$
\int_{B\left(z^{\prime}, 2 r_{1}\right)} u(z) d m(z)=-\infty,
$$

which implies as before $u\left(z^{\prime}\right)=-\infty$. So $\Omega_{0}$, the interior of the set $\{z \in \Omega: u(z)=-\infty\}$, is a nonempty open set. If $z$ is a limit point of $\Omega_{0}$ in $\Omega$, then by the above argument, we also have $u(z)=-\infty$ and $z \in \Omega_{0}$; hence $\Omega_{0}$ is also closed in $\Omega$. Since $\Omega$ is connected, $\Omega_{0}=\Omega$ and $u \equiv-\infty$, a contradiction. Q.E.D.

If $f$ is a holomorphic function in $\Omega$, then $\log |f|$ is a plurisubharmonic function in $\Omega$. Thus we have the following corollary which will be used in the proof of the Blaschke condition. 
COROllaRy 5.3. Let $\Omega=U^{N}$ or $B_{N}$ and let $m$ be the Lebesgue measure on $\Omega$ normalized so that $m(\Omega)=1$. Let $f \in H(\Omega), f \not \equiv 0$. Then

$$
\int_{\Omega} \log |f(r z)| d m(z)>-\infty \quad \text { if } 0<r \leqq 1
$$

If $f(0) \neq 0$, then

$$
\int_{\Omega} \log |f(z)| d m(z) \geqq \log |f(0)| .
$$

If $f \in H^{\infty}(\Omega)$, then $\log |f| \in L^{1}(m)$.

6. The Blaschke condition. We now show that the generalized Blaschke condition holds for bounded holomorphic functions in several complex variables. We begin with a (well-known) lemma.

Lemma 6.1. Let $X$ be a Lebesgue measurable subset of $\boldsymbol{R}^{N}, I$ an interval in $\boldsymbol{R}$. For each positive integer $k$, let $m_{k}$ be the Lebesgue measure on $\boldsymbol{R}^{k}$. Let $f: I \times X$ $\rightarrow[-\infty, \infty]$ be a function satisfying the conditions

(i) for each $t \in I, x \rightarrow f(t, x)$ is Lebesgue measurable,

(ii) for each $x \in X, t \rightarrow f(t, x)$ is increasing.

Then $f$ is a Lebesgue measurable function in $I \times X$.

Proof. In what follows, measurable will mean Lebesgue measurable. It is sufficient to prove that

$$
A=\{(t, x) \in I \times X: f(t, x)>\alpha\}
$$

is measurable for every real number $\alpha$. Since $I$ is $\sigma$-compact and $X$ is the union of an $F_{\sigma}$ and a set of measure zero, we may assume that they are compact.

Let $\varepsilon>0$ be given. Choose points $t_{0}, t_{1}, \ldots, t_{n} \in I$ such that $t_{0}<t_{1}<\cdots<t_{n}$, $\left[t_{0}, t_{n}\right]=I$ and $m_{1}\left(I_{i}\right) \leqq \varepsilon$ where $I_{i}=\left[t_{i}, t_{i+1}\right], 0 \leqq i \leqq n-1$. Let $A_{i}=\left\{x: f\left(t_{i}, x\right)>\alpha\right\}$. By condition (i), each $A_{i}$ is measurable. By (ii), $A_{i} \subseteq A_{j}$ if $i \leqq j$. Let $B=\bigcup_{i=0}^{n-1}\left(I_{i} \times A_{i}\right)$, $C=\bigcup_{i=0}^{n-1}\left(I_{i} \times A_{i+1}\right)$. Then $B$ and $C$ are measurable subsets of $I \times X$ and by the condition (ii), it is easy to check that $B \subseteq A \subseteq C$. Now

$$
C-B=\bigcup_{i=0}^{n-1}\left(I_{i} \times\left(A_{i+1}-A_{i}\right)\right) .
$$

Since $\left(A_{i+1}-A_{i}\right) \cap\left(A_{i}-A_{i-1}\right)=\varnothing$ for all $i$, we have

$$
\begin{aligned}
m_{N+1}(C-B) & =\sum_{i=0}^{n-1} m_{1}\left(I_{i}\right) m_{N}\left(A_{i+1}-A_{i}\right) \\
& \leqq \varepsilon \sum_{i=0}^{n-1} m_{N}\left(A_{i+1}-A_{i}\right) \\
& =\varepsilon m_{N}\left(\bigcup_{i=0}^{n-1}\left(A_{i+1}-A_{i}\right)\right) \\
& \leqq \varepsilon m_{N}(X) .
\end{aligned}
$$


Let $\varepsilon=1, \frac{1}{2}, \frac{1}{3}, \ldots$. Then we see that there is an increasing sequence $B_{k}$ and a decreasing sequence $C_{k}$ of measurable sets such that with $E=\bigcup_{k=1}^{\infty} B_{k}, F=$ $\bigcup_{k=1}^{\infty} C_{k}$, we have $E \subseteq A \subseteq F$ and $m_{N+1}(F-E)=0$. Hence $A$ is Lebesgue measurable.

For our application, we note that the Lebesgue measure in $\boldsymbol{R}^{N}$ coincides with the Hausdorff measure $H_{N}$ in $\boldsymbol{R}^{N}$. For what follows, we shall use the following notation: $N$ will denote a positive integer. For $j=1,2, \ldots, N+1, \pi_{j}$ will denote the projection on $\boldsymbol{C}^{N+1}$ defined by

$$
\pi_{j}(z)=\left(z_{1}, \ldots, z_{j-1}, 0, z_{j+1}, \ldots, z_{N+1}\right)
$$

and we write $\hat{z}_{j}=\pi_{j}(z) . \Omega$ will denote $U^{N+1}$ or $B_{N+1}$ and for $0<r<1, \Omega(r)$ is the corresponding domain of radius $r$.

LEMMA 6.2. Let $f \in H(\Omega), f \not \equiv$ and $\mu$ its multiplicity function. Let $V_{r}=Z(f)$ $\cap \bar{\Omega}(r)$. Then for each $j, 1 \leqq j \leqq N+1$, the function

$$
F\left(r, \hat{z}_{j}\right)=\int_{V_{r} \cap \pi_{j}^{-1}\left(\hat{z}_{j}\right)} \mu(z) d H_{0}\left(z_{j}\right)
$$

is Lebesgue measurable in $(0,1) \times \pi_{j} \Omega$.

Proof. Fix $j, 1 \leqq j \leqq N+1$. Clearly $F\left(r, \hat{z}_{j}\right)$ is an increasing function of $r$ for each $\hat{z}_{j}$. Thus in view of Lemma 6.1 , we need only show that $F_{r}: \hat{z}_{j} \rightarrow F\left(r, \hat{z}_{j}\right)$ is Lebesgue measurable for each $r$.

Fix $r \in(0,1)$. Let $S$ be the singular locus of $Z(f)$. Then $H_{2 N}(S)=0$; hence by property (ii) of the Hausdorff measures, $H_{2 N}\left(\pi_{j} S\right)=0$. We shall show that $F_{r}$ is a Borel function on $R=\pi, \Omega-\pi_{j} S$. This will imply that $F_{r}$ is Lebesgue measurable on $\pi_{j} \Omega$.

The value $n=F\left(r, \hat{z}_{j}\right)$ is a nonnegative integer or $\infty$. Suppose $n \neq 0$ or $\infty$. Then $V_{r} \cap \pi_{j}^{-1}\left(\hat{z}_{j}\right)$ consists of only a finite number of points. If $\hat{z}_{j} \in R$, then each point of $V_{r} \cap \pi_{j}^{-1}\left(\hat{z}_{j}\right)$ is a regular point of $Z(f)$. By Proposition 4.1, $\mu(z)$ is constant in a neighborhood of each such point. So $F_{r}$ is constant in a neighborhood of $\hat{z}_{j}$. If $n=0$, then $f$ has no zeros on $V_{r} \cap \pi_{j}^{-1}\left(\hat{z}_{j}\right)$. By the continuity of $f$, it has no zeros in a neighborhood of $V_{r} \cap \pi_{j}^{-1}\left(\hat{z}_{j}\right)$. Thus for each integer $n, 0 \leqq n<\infty$, the set $A_{n}=\left\{\hat{z}_{j} \in R: F\left(r, \hat{z}_{j}\right)=n\right\}$ is an open set in $R$. Since $A_{\infty}=\left\{\hat{z}_{j} \in R: F\left(r, \hat{z}_{j}\right)=\infty\right\}$ $=R-\bigcup_{n=1}^{\infty} A_{n}$, we see that $A_{\infty}$ is a closed set of $R$. This shows that $F_{r}$ is a Borel function on $R$ and the proof is complete.

THEOREM 6.3. Let $f \in H^{\infty}(\Omega), f \not \equiv 0$ and $|f| \leqq 1$. Let $\mu$ be its multiplicity function. Then

$$
\int_{0}^{1} d r \int_{\bar{\Omega}(r)} \mu(z) d H_{2 N}(z)<\infty
$$

If $f(0) \neq 0$, then

$$
\int_{0}^{1} d r \int_{\bar{\Omega}(r)} \mu(z) d H_{2 N}(z) \leqq c(\Omega) \log \frac{1}{|f(0)|}
$$

where $c\left(U^{N+1}\right)=(N+1) \pi^{N}, c\left(B_{N+1}\right)=(N+1) \pi^{N} / N !$. 
Proof. Assume first that $f\left(\hat{z}_{j}\right) \neq 0$ for all $j, 1 \leqq j \leqq N+1$. Let $f_{z_{j}}\left(z_{j}\right)=f(z)$ and let $\mu_{z_{j}}\left(z_{j}\right)$ be the zero-multiplicity of $f_{z_{j}}$ at $z_{j}$. It is easily seen that $\mu_{i_{j}}\left(z_{j}\right) \geqq \mu(z)$. Let $n_{z,}(r)$ be the number of zeros of $f_{\hat{z}}$ in $\pi_{j}^{-1}\left(\hat{z}_{j}\right) \cap \bar{\Omega}(r)$, counting multiplicities. Let $V_{r}=Z(f) \cap \bar{\Omega}(r)$. By Corollary 4.3,

$$
\int_{\bar{\Omega}(r)} \mu(z) d H_{2 N}(z)=\sum_{j=1}^{N+1} \int_{\pi_{j} \bar{\Omega}(r)} d H_{2 N}\left(\hat{z}_{j}\right) \int_{\bar{\Omega}_{(r) \cap \pi_{j}^{-1}\left(\hat{z}_{j}\right)}} \mu(z) d H_{0}\left(z_{j}\right) .
$$

Since $\hat{z}_{j} \notin \pi_{j} \bar{\Omega}(r)$ implies $V_{r} \cap \pi_{j}^{-1}\left(\hat{z}_{j}\right)=\varnothing$, this can be written

$$
\begin{aligned}
\int_{\bar{\Omega}(r)} \mu(z) d H_{2 N}(z) & =\sum_{j=1}^{N+1} \int_{\pi_{j} \Omega} d H_{2 N}\left(\hat{z}_{j}\right) \int_{V_{r} \cap \pi_{j}^{-1}\left(\hat{z}_{j}\right)} \mu(z) d H_{0}\left(z_{j}\right) \\
& =\sum_{j=1}^{N+1} \int_{\pi_{j} \Omega} F\left(r, \hat{z}_{j}\right) d H_{2 N}\left(\hat{z}_{j}\right)
\end{aligned}
$$

where

$$
F\left(r, \hat{z}_{j}\right)=\int_{V_{r} \cap \pi_{j}^{-1}\left(\hat{z}_{j}\right)} \mu(z) d H_{0}\left(z_{i}\right) .
$$

By Lemma 6.2, $F$ is Lebesgue measurable in $(0,1) \times \pi_{j} \Omega$ and Fubini's theorem applies to give

$$
\int_{0}^{1} d r \int_{\pi_{j} \Omega} F\left(r, \hat{z}_{j}\right) d H_{2 N}\left(\hat{z}_{j}\right)=\int_{\pi_{j} \Omega} d H_{2 N}\left(\hat{z}_{j}\right) \int_{0}^{1} F\left(r, \hat{z}_{j}\right) d r .
$$

Since $\mu(z) \leqq \mu_{\hat{z}_{j}}\left(z_{j}\right)$, we have

$$
F\left(r, \hat{z}_{j}\right) \leqq \int_{V_{r} \cap \pi_{j}^{-1}\left(\hat{z}_{j}\right)} \mu_{j \hat{z}}\left(z_{j}\right) d H_{0}\left(z_{j}\right)=n_{\dot{z}_{j}}(\rho)
$$

where $\rho=r$ if $\Omega=U^{N+1}$ and $\rho=\left(r^{2}-\left\|\hat{z}_{j}\right\|^{2}\right)^{1 / 2}$ if $\Omega=B_{N+1}\left(\left\|\hat{z}_{j}\right\|\right.$ is Euclidean norm of $\hat{z}_{j}$ ). Noting that $d r / d \rho=\rho / r \leqq 1$, we get by Jensen's formula (2),

$$
\int_{0}^{1} n_{z_{j}}(\rho) d r=\int_{0}^{1} n_{\varepsilon_{j}}(r) d r \leqq \log \frac{1}{\left|f\left(\hat{z}_{j}\right)\right|} \quad \text { if } \Omega=U^{N+1}
$$

and

$$
\begin{aligned}
\int_{0}^{1} n_{z_{j}}(\rho) d r & =\int_{0}^{a} n_{z_{j}}(\rho) \frac{\rho}{r} d \rho \leqq \int_{0}^{a} n_{z_{j}}(\rho) d \rho, \quad\left(a=\left(1-\left\|\hat{z}_{j}\right\|^{2}\right)^{1 / 2}\right) \\
& \leqq \log \frac{1}{\left|f\left(\hat{z}_{j}\right)\right|} \quad \text { if } \Omega=B_{N+1}
\end{aligned}
$$

Thus

$$
\int_{0}^{1} F\left(r, \hat{z}_{j}\right) d r \leqq \log \frac{1}{\left|f\left(\hat{z}_{j}\right)\right|}
$$

Integratirig (19) with respect to $r$ and substituting (20) and (21), we get

$$
\int_{0}^{1} d r \int_{\bar{\Omega}(r)} \mu(z) d H_{2 N}(z) \leqq \sum_{j=1}^{N+1} \int_{\pi, \Omega} \log \frac{1}{\left|f\left(\hat{z}_{j}\right)\right|} d H_{2 N}\left(\hat{z}_{j}\right) .
$$

Since $f\left(\hat{z}_{j}\right) \not \equiv 0$ for all $j$, Corollary 5.3 shows that each integral on the right is finite and (17) is proved. 
If $f(0) \neq 0$, Corollary 5.3 gives

Hence,

$$
\int_{\pi, \Omega} \log \frac{1}{\left|f\left(\hat{z}_{j}\right)\right|} d H_{2 N}\left(\hat{z}_{j}\right) \leqq H_{2 N}\left(\pi_{j} \Omega\right) \log \frac{1}{|f(0)|} .
$$

$$
\int_{0}^{1} d r \int_{\bar{\Omega}(r)} \mu(z) d H_{2 N}(z) \leqq\left\{\sum_{j=1}^{N+1} H_{2 N}\left(\pi_{j} \Omega\right)\right\} \log \frac{1}{|f(0)|}
$$

Putting $c(\Omega)=\sum_{j=1}^{N+1} H_{2 N}\left(\pi_{j} \Omega\right)$, we get (18).

The case when $f\left(\hat{z}_{j}\right) \equiv 0$ for some $j$ can be reduced to the first case as follows. We do this separately for $U^{N+1}$ and $B_{N+1}$.

For $U^{N+1}$. If $f\left(\hat{z}_{j}\right) \equiv 0$ for some $j$, then there is a positive integer $\alpha_{j}$ such that $g_{j}(z)=f(z) / z_{j}^{\alpha_{j}}$ is holomorphic in $U^{N+1}$ and $g_{j}\left(\hat{z}_{j}\right) \neq \equiv$. Doing this for all $j$, we get nonnegative integers $\alpha_{j}$ such that

$$
f(z)=z_{1}^{\alpha_{1}} \cdots z_{N+1}^{\alpha_{N+1}} g(z)
$$

where $g$ is holomorphic in $U^{N+1}$ and $g\left(\hat{z}_{j}\right) \not \equiv 0$ for all $j$. Since $|f(z)| \leqq 1$ as $z$ tends to $T^{N+1}$, the same is true for $g$, so that $|g| \leqq 1$ in $U^{N+1}$ by the maximum modulus theorem. Thus the first part of the proof applies to $g$. An easy computation shows that each factor $z_{j}^{\alpha_{j}}$ contributes $\alpha_{j} \pi^{N} /(2 N+1)$ to the integral in (17). Thus with $\mu_{g}=$ multiplicity function of $g$,

$$
\int_{0}^{1} d r \int_{\bar{\Omega}(r)} \mu(z) d H_{2 N}(z)=\int_{0}^{1} d r \int_{\bar{\Omega}(r)} \mu_{g}(z) d H_{2 N}(z)+\frac{\pi^{N}}{2 N+1}\left(\sum_{1}^{N+1} \alpha_{j}\right)<\infty .
$$

For $B_{N+1}$, we have the following lemma.

Lemma 6.4. Let $f \in H\left(B_{N}\right), f \not \equiv 0$. Then there exists a coordinate system $z_{1}, \ldots, z_{N}$ such that $f\left(\hat{z}_{j}\right) \not \equiv 0$ for all $j$.

Proof. Without loss of generality, we may assume that $f \in H\left(\bar{B}_{N}\right)$. Let $e_{1}, \ldots, e_{N}$ be $N$ points on $S^{2 N-1}$ which form an orthogonal basis for $C^{N}$. We shall show that there exists a unitary transformation $A$ such that $f\left(A e_{1}\right) f\left(A e_{2}\right) \cdots f\left(A e_{N}\right) \neq 0$. Then $A e_{1}, \ldots, A e_{N}$ will give the required coordinate system.

If $f\left(e_{1}\right) \neq 0$, we take $A_{1}=I$ the identity transformation. If $f\left(e_{1}\right)=0$, then since $f$ is not identically zero on $S^{2 N-1}$, there is an $\tilde{e}_{1} \in S^{2 N-1}$ such that $f\left(\tilde{e}_{1}\right) \neq 0$. Since the unitary transformations are transitive on $S^{2 N-1}$, we can find a unitary transformation $A_{1}$ such that $A_{1} e_{1}=\tilde{e}_{1}$. By the continuity of $f$, there is a neighborhood $W_{1}$ of $\tilde{e}_{1}$ such that $f(e) \neq 0$ for all $e \in W_{1}$.

If $f\left(A_{1} e_{2}\right) \neq 0$, we take $A_{2}=I$. If $f\left(A_{1} e_{2}\right)=0$, then since $Z(f)$ is nowhere dense on $S^{2 N-1}$, there is an $\tilde{e}_{2}$ arbitrarily close to $A_{1} e_{2}$ such that $f\left(\tilde{e}_{2}\right) \neq 0$. Then $\tilde{e}_{2}=A_{2} A_{1} e_{2}$ for some unitary transformation $A_{2}$. We can choose $\tilde{e}_{2}$ so close to $A_{1} e_{2}$ that $A_{2} \tilde{e}_{1}$ $\in W_{1}$. Fix such an $A_{2}$. Then there exists a neighborhood $W_{2}$ of $\tilde{e}_{2}$ such that $f(e) \neq 0$ for all $e \in W_{2}$.

Continuing the process $N$ times, we get unitary transformations $A_{1}, \ldots, A_{N}$ such that if $A=A_{N} A_{N-1}, \ldots, A_{1}$, then $f\left(A e_{j}\right) \neq 0$ for all $j$. This completes the proof of the lemma and that of the theorem. 
7. Examples. In contrast to the theorem in one variable, the Blaschke condition is not sufficient for an analytic subvariety to be the zero-set of a bounded holomorphic function in $U^{2}$ or $B_{2}$. In fact there are analytic subvarieties which satisfy the Blaschke condition and which are determining sets for bounded holomorphic functions.

EXAMPLE 1. Let $\alpha_{n}=1-1 / n$ and

$$
V=\left\{\left(\alpha_{n}, w\right):\left|\alpha_{n}\right|^{2}+|w|^{2}<1, n=1,2,3, \ldots\right\} .
$$

Then (by Cartan's Theorem B) $V$ is the zero-set of a holomorphic function in $\boldsymbol{B}_{2}$. But $V$ is a $D$-set for bounded holomorphic functions in $B_{2}$, although it satisfies the Blaschke condition.

An easy calculation shows that $H_{2}\left(V_{r}\right)=\pi \sum_{\left|\alpha_{n}\right| \leqq r}\left(r^{2}-\alpha_{n}^{2}\right)$. Hence

$$
\begin{aligned}
\int_{0}^{1} H_{2}\left(V_{r}\right) d r & =\pi \int_{0}^{1} \sum_{\left|\alpha_{n}\right| \leqq r}\left(r^{2}-\alpha_{n}^{2}\right) d r=\pi \sum_{n=1}^{\infty} \int_{\left|\alpha_{n}\right|}^{1}\left(r^{2}-\alpha_{n}^{2}\right) d r \\
& =\frac{\pi}{3} \sum_{n=1}^{\infty}\left(1-\alpha_{n}\right)^{2}\left(1+2 \alpha_{n}\right)=\frac{\pi}{3} \sum_{n=1}^{\infty} \frac{1}{n^{2}}\left(3-\frac{2}{n}\right)<\infty .
\end{aligned}
$$

Now suppose $f \in H^{\infty}\left(B_{2}\right)$ and $f=0$ on $V$. We shall show that then $f \equiv 0$.

For each $c \in C$, let $D(c)$ be the disc in the $z$-plane passing through the point $z=1$ and having center at $z=|c|^{2} /\left(1+|c|^{2}\right) . D(c) \subset U$ for all $c$. Let

$$
P(c)=\{(z, c(1-z)): z \in D(c)\} \text {. }
$$

Then $P(c)$ is a disc imbedded in $B_{2}$ and its boundary passes through the point $(1,0)$ for all $c$.

For each $c$, let $f_{c}(z)=f(z, c(1-z)), z \in D(c)$. When $n$ is sufficiently large, $\alpha_{n} \in D(c)$ and $f_{c}\left(\alpha_{n}\right)=0$. Therefore the zero-set of $f_{c}$ violates the Blaschke condition. Since $f_{c}$ is bounded, $f_{c} \equiv 0$, i.e. $\left.f\right|_{P(c)} \equiv 0$ for all $c$. Since $B_{2}=\bigcup_{c \in C} P(c)$, we have $f \equiv 0$.

EXAMPLE 2. Fix $\delta, \frac{1}{2}<\delta<1$. Let $\alpha_{n}=1-1 / n^{\delta}$ and

$$
V=\left\{\left(z, 2 \alpha_{n}-z\right):|z|<1,\left|2 \alpha_{n}-z\right|<1, n=1,2,3, \ldots\right\} .
$$

Then $V$ is the zero-set of a holomorphic function in $U^{2}$. We shall show that it satisfies the Blaschke condition and is a $D$-set for bounded holomorphic functions.

For each $n$, the area of the set $\left\{\left(z, 2 \alpha_{n}-z\right):|z| \leqq r,\left|2 \alpha_{n}-z\right| \leqq r\right\}$ is $\leqq 2 \pi\left(r^{2}-\alpha_{n}^{2}\right)$. So $H_{2}\left(V_{r}\right) \leqq 2 \pi \sum_{\left|\alpha_{n}\right| \leqq r}\left(r^{2}-\alpha_{n}^{2}\right)$. The computation in Example 1 shows that

$$
\begin{aligned}
\int_{0}^{1} H_{2}\left(V_{r}\right) d r & \leqq \frac{2 \pi}{3} \sum_{n=1}^{\infty}\left(1-\alpha_{n}\right)^{2}\left(1+2 \alpha_{n}\right) \\
& =\frac{2 \pi}{3} \sum_{n=1}^{\infty} \frac{1}{n^{2 \delta}}\left(1+2 \alpha_{n}\right) \\
& <\infty \quad \text { since } 2 \delta>1 .
\end{aligned}
$$

Let $f \in H^{\infty}\left(U^{2}\right)$ and $f=0$ on $V$. Let

$$
A=\{c \in C: \operatorname{Re} c>1,|\arg c|<(1-\delta) \pi / 2\} .
$$


For $c \in A$, the boundary of the disc $U(c)$ of radius $1 /|c|$ and center at $1-1 / c$ makes an angle $k \pi / 2$ with the real axis, where $\delta<k<1$. The real axis divides $U(c)$ into two regions; let $U_{1}(c)$ be the smaller one. Let $D(c)$ be the region formed by $U_{1}(c)$ and its reflection in the real axis. Then $D(c)$ is contained in the unit disc $U$ and is bounded by two circular arcs meeting at an angle $k \pi$ at the point $z=1$ and $z=z_{0}$, where $z_{0}=1-2 \operatorname{Re} c /|c|^{2}$. Let $P(c)=\{(z, c(z-1)+1): z \in D(c)\}$. For every $c \in A, P(c)$ is a subset of $U^{2}$ such that the point $(1,1)$ lies on its boundary.

Fix $c \in A$. Define $f_{c}(z)=f(z, c(z-1)+1), z \in D(c)$. Let $\tilde{\alpha}_{n}=1-(2 /(1+c))\left(1 / n^{\delta}\right)$. For all sufficiently large $n, \tilde{\alpha}_{n} \in D(c)$ and since $f=0$ on $V, f_{c}\left(\tilde{\alpha}_{n}\right)=0$. Under the mapping $\varphi_{c}(z)=\left((1-z) /\left(z-z_{0}\right)\right)^{1 / k}, D(c)$ is mapped onto the right half-plane. Let $\beta_{n}=\varphi_{c}\left(\tilde{\alpha}_{n}\right)$. Then it is easy to check that for fixed $c \in A, \operatorname{Re} \beta_{n} \geqq \gamma n^{-\delta / k}$ for sufficiently large $n$, where $\gamma$ is positive and does not depend on $n$. Since $\delta / k<1$, it follows that

$$
\sum \operatorname{Re} \beta_{n}=\infty \text {. }
$$

Thus the function $\tilde{f}_{c}=f_{c} \cdot \varphi_{c}^{-1}$ is a bounded holomorphic function in the right half-plane whose zeros $\beta_{n}$ satisfy (22). So $f_{c} \equiv 0$ which implies that $f_{c} \equiv 0$, i.e. $\left.f\right|_{P(c)} \equiv 0$ for all $c \in A$. Let $P=\bigcup_{c \in A} P(c)$. Then $P$ contains an open subset of $U^{2}$ since the open subset $D \times \Delta$ of $C^{2}$, where $D=D(1+i)$ and $\Delta=A \cap\{c:|c-1|<1\}$, is mapped into $P$ by $\Phi:(z, c) \rightarrow(z, c(z-1)+1)$ which is nonsingular when $z \neq 1$. So $f=0$ on $P$ implies $f \equiv 0$ in $U^{2}$.

Added in proof. Recently the author has extended Theorem 6.3 to wider classes of functions, namely the Nevanlinna classes on $U^{N}$ and $B^{N}$.

\section{REFERENCES}

1. A. C. Aitken, Determinants and matrices, 9th ed., Oliver and Boyd, Edinburgh, 1956.

2. S. Bochner, Classes of holomorphic functions of several variables in circular domains, Proc. Nat. Acad. Sci. U.S.A. 46 (1960), 721-723. MR 22 \#11144.

3. H. Federer, Surface area. II, Trans. Amer. Math. Soc. 55 (1944), 438-456. MR 6, 45.

4. R. C. Gunning and H. Rossi, Analytic functions of several complex variables, PrenticeHall, Englewood Cliffs, N. J., 1965. MR 31 \#4927.

5. P. R. Halmos, Measure theory, Van Nostrand, Princeton, N. J., 1950. MR 11, 504.

6. W. Rudin, Function theory in polydiscs, Math. Lecture Notes, Benjamin, New York, 1969.

7. - Real and complex analysis, McGraw-Hill, New York, 1966. MR 35 \#1420.

8. L. Schwartz, Cours d'analysis. I, Hermann, Paris, 1967.

9. G. Stolzenberg, Volumes, limits and extensions of analytic varieties, Lecture Notes in Math., no. 19, Springer-Verlag, Berlin, 1966. MR 34 \#6156.

10. E. L. Stout, The second cousin problem with bounded data, Pacific J. Math. 26 (1968), 379-387.

11. E. C. Titchmarsh, The theory of functions, 2nd ed., Oxford Univ. Press, London, 1939.

12. V. S. Vladimirov, Methods of the theory of functions of several complex variables, "Nauka", Moscow, 1964; English transl., M.I.T. Press, Cambridge, Mass., 1966. MR 30 \#2163; MR 34 \#1551.

UNIVERSITY OF WISCONSIN

MADISON, WISCONSIN 